\title{
Robert Belot et Pierre Lamard, Peugeot à Sochaux, des hommes une usine, un territoire
}

Panazol, Lavauzelle, 2007, 372 pages.

Michel Cotte

\section{OpenEdition}

Journals

Édition électronique

URL : http://journals.openedition.org/dht/1081

DOI : 10.4000/dht.1081

ISSN : $1775-4194$

Éditeur :

Centre d'histoire des techniques et de l'environnement du Cnam (CDHTE-Cnam), Société des élèves du CDHTE-Cnam

\section{Édition imprimée}

Date de publication : 1 juin 2008

Pagination : 236-238

ISBN : 978-2-95-30779-1-9

ISSN : 0417-8726

Référence électronique

Michel Cotte, "Robert Belot et Pierre Lamard, Peugeot à Sochaux, des hommes une usine, un territoire », Documents pour I'histoire des techniques [En ligne], 15 | $7^{\text {er }}$ semestre 2008, mis en ligne le 22 octobre 2010, consulté le 24 septembre 2020. URL : http://journals.openedition.org/dht/1081 ; DOl : https:// doi.org/10.4000/dht.1081

Ce document a été généré automatiquement le 24 septembre 2020

(C) Tous droits réservés 


\title{
Robert Belot et Pierre Lamard, Peugeot à Sochaux, des hommes une usine, un territoire
}

Panazol, Lavauzelle, 2007, 372 pages.

\author{
Michel Cotte
}

\section{RÉFÉRENCE}

Robert Belot et Pierre Lamard, Peugeot à Sochaux, des hommes une usine, un territoire, Panazol, Lavauzelle, 2007, 372 pages.

1 Les auteurs sont tous deux professeurs à l'Université de technologie de BelfortMontbéliard, où ils animent simultanément le laboratoire RECITS (Recherches et études sur les choix industriels, techniques et scientifiques) et le département des Humanités de cette belle école d'ingénieur, qui a compris tout l'intérêt des sciences humaines et sociales pour la formation de ses élèves. C'est donc en voisins, mais aussi en spécialistes des questions d'histoire liées à l'industrialisation, à ses aménagements, à ses hommes et à ses territoires, qu'ils nous apportent cette très belle étude d'ensemble d'un site emblématique de l'industrie française du $\mathrm{XX}^{\mathrm{e}}$ siècle.

Longtemps lieu principal du développement des usines Peugeot, le cœur de la firme au Lion a bien battu là, à Sochaux, près de 90 ans, et il reste aujourd'hui encore l'une des "plateformes" essentielles du groupe PSA. Sochaux a donné un lieu exceptionnel à l'industrie française, ancré dans un "pays" de vieille tradition technique et industrielle, la région de Montbéliard. Sochaux est bien une certaine manière de concevoir la production automobile et de l'organiser en un véritable système social et industriel, à l'échelle d'un territoire. Depuis la fin de la Grande Guerre, le site a rassemblé un outil longtemps unique en France par son étendue et la rationalité de ses fonctions productives. Il compta jusqu'à 40000 ouvriers dans les années 1970. 
3 Robert Belot et Pierre Lamard nous offrent un ouvrage des plus complets à propos de ce site majeur. Ils en montrent parfaitement la continuité historique comme ses multiples dimensions techniques, sociales et territoriales. Non seulement ils rassemblent une iconographie exceptionnelle, presque entièrement inédite et provenant directement des archives de l'usine, mais ils présentent un texte très bien structuré, impeccablement documenté et parfaitement maîtrisé dans la mise en perspective des multiples facettes d'une histoire industrielle globale. En outre, l'harmonie du texte et des images est de très haute qualité, dans un grand format bienvenu qui rend particulièrement agréable et féconde la consultation de ce beau livre. Il faut souligner la réussite des deux auteurs dans cet équilibre rarement atteint, surtout quand il s'agit d'automobile. L'empathie qu'ils éprouvent pour leur sujet ne les empêche en rien de garder la distance et la lucidité nécessaires à une œuvre d'histoire pleinement achevée.

4 La première partie, "Travailler ensemble», commence par la mise à plat de l'organisation sociale du site. Nous rencontrons les dirigeants dont sut s'entourer la famille Peugeot, à commencer par Ernest Mattern, l'homme qui fit de Sochaux un laboratoire de l'organisation du travail. C'est sous son impulsion, autour de la forge, lieu sochalien mythique entre tous, que la 201 lança la production de masse de la voiture automobile en France, sur un modèle productif véritablement fordien (1929). Les ingénieurs, les cadres, les contremaîtres et les différents groupes de techniciens ont toujours joué un rôle clé dans l'usine, formant de véritables archétypes où culminent les notions de mérite, de fidélité, de dévouement à une culture industrielle. Dans une formule heureuse, les ouvriers sont de véritables « soldats de la modernité ", et ils sont présentés dans leur corps à corps avec la machine. Les photos sont là particulièrement évocatrices, alors que le texte explicite les problématiques de la main d'œuvre et du travail posté, dans la continuité historique.

5 Les «identités parallèles » montrent tout d'abord le rôle des femmes dans la grande industrie automobile. Cette question originale et sensible agit comme un révélateur de l'écart entre les réalités du travail quotidien et les représentations sociales exacerbées de la modernité automobile du $\mathrm{XX}^{\mathrm{e}}$ siècle. La tradition des travailleurs immigrés traverse elle, de manière majeure, toute l'histoire de cette industrie, tout particulièrement à Sochaux où ils sont présents dès l'ouverture du site, pendant la guerre, justement au côté des femmes... Ce sont eux qui permettent de gagner la bataille de l'intensification de la production des années 1960-70.

6 La formation des apprentis, des ouvriers et des techniciens est elle aussi magnifiquement illustrée et commentée. Elle s'effectua longtemps dans des ateliersécoles au sein de l'usine, et il y eut même une importante "école des cadres " sochalienne, mais elle s'orienta ensuite vers des partenariats extérieurs. La communication interne de l'entreprise apporte un chapitre important pour la compréhension de la culture industrielle propre au monde de Sochaux, de ses thèmes majeurs qui marquèrent les différentes époques. Elle contribua au sentiment d'une forte identité collective.

7 L'ouvrage aborde ensuite l'histoire de la production, dans ce « combat toujours recommencé de la modernisation». Ce chapitre s'attache à décrire l'adaptation continue de la production de masse faite au moyen de la chaîne fordienne, et à en comprendre les évolutions techniques, les choix organisationnels successifs, les investissements souvent décisifs dans le parc des machines-outils. La direction des études et ses laboratoires d'essais sont également mis en valeur, par leur rôle au service 
de la conception des véhicules et de la qualité de la production. Une part importante est consacrée au Centre de Belchamp qui, à partir de 1959, dans un vaste un site forestier proche de Sochaux, permit le développement d'un outil très complet d'essais et d'études essentiel à l'ensemble des usines Peugeot, puis du groupe PSA, avec piste d'essais, anneau de vitesse, "crash-test », etc. La métrologie, le calcul et le traitement de l'information qui complètent l'arsenal industriel de la conception et de la production font l'objet d'un chapitre spécifique.

Ne voulant pas tomber dans la fascination pour l'objet automobile, qui est le travers le plus fréquent des ouvrages sur cette industrie phare du $\mathrm{XX}^{\mathrm{e}}$ siècle occidental, les auteurs ont là carrément fait court : seulement trois modèles sont présentés en moins de 15 pages, pour un petit siècle de production. Chacun illustre une étape jugée charnière de l'histoire de Sochaux. Il s'agit de la 201 dans les années 30, qui lance la production de masse ; puis la 203 et de la renaissance industrielle des années 50 ; enfin la 307 , en fleuron de la modernité et du design des années 2000 de la firme au Lion... Sans trop alourdir cette partie, un ou deux modèles complémentaires auraient pu retenir l'attention des auteurs, car la grande période productive allant des années 1960 aux années 1980 n'est pas présente, et c'est un peu dommage au sein d'un ouvrage par ailleurs si complet et si bien documenté. Une place importante est ensuite réservée à ce qui fut longtemps la publicité de l'entreprise: faire connaître et faire rêver ! Un véritable mythe de Sochaux s'élabore auprès des concessionnaires et des clients, illustrant les représentations successives de la modernité automobile sous l'emblème du Lion.

9 Les auteurs se penchent ensuite, avec beaucoup de pertinence, sur la construction de l'espace et sur sa gestion industrielle, à propos de cet exemple exceptionnel de Sochaux, longtemps considéré comme la plus grande usine de France. C'est un site unique et continu, mais il est en fait composé d'un ensemble d'usines complémentaires sans cesse recomposées. Sochaux est un perpétuel chantier depuis ses origines, de quoi alimenter la réflexion patrimoniale si elle se contente uniquement de préservation architecturale! Il faut souligner la complétude et le haut niveau de cette importante partie du livre, en pleine continuité avec les problématiques actuelles du site: les relations à l'eau et à l'environnement, la sécurité industrielle, la mobilité et les transports de la "ruche sochalienne ", l'urbanisme industriel, le système économique organisé autour de l'entreprise, etc.

10 Cela nous conduit à la dernière partie de l'ouvrage "Vivre son siècle " qui aborde en premier lieu les impacts de la guerre, là encore un chapitre parfaitement documenté et remarquablement illustré. Les conflits sociaux et la vie syndicale sont ensuite présentés, apportant une analyse approfondie du modèle social sochalien et de son "vivre ensemble ». Le sport y tient une place importante et la construction du Stade de la forge (1929-30), au sein même de l'usine, devenu ensuite Stade Bonal du nom d'un de ses directeurs, résistant mort en déportation, résume cette partie.

11 Il nous faut remercier les auteurs pour ce très bel ouvrage de référence, en matière d'étude et de mise en perspective historique d'un site industriel. 


\section{AUTEURS}

MICHEL COTTE

Centre François Viète, Université de Nantes 